\title{
Mice lacking microRNAs in Pax8-expressing cells develop hypothyroidism and end-stage renal failure
}

\author{
Malte P. Bartram¹ , Elena Amendola², Thomas Benzing ${ }^{1,3,4}$, Bernhard Schermer ${ }^{1,3,4}$, Gabriella de Vita ${ }^{2}$ \\ and Roman-Ulrich Müller ${ }^{1,3,4^{*}}$
}

\begin{abstract}
Background: Non-coding RNAs have gained increasing attention during the last decade. The first large group of non-coding RNAs to be characterized systematically starting at the beginning of the 21st century were small oligonucleotides - the so-called microRNAs (miRNAs). By now we have learnt that microRNAs are indispensable for most biological processes including organogenesis and maintenance of organ structure and function. The role of microRNAs has been studied extensively in the development of a number of organs, so far most studies focussed on e.g. the heart or the brain whilst the role of microRNAs in the development and maintenance of complex epithelial organs is less well understood. Furthermore most analyses regarding microRNA function in epithelial organs employed conditional knockout mouse models of the RNAse III Dicer to abrogate microRNA biogenesis. However, there is increasing evidence for Dicer to have multiple functions independent from microRNA maturation. Therefore Dicer independent models are needed to gain further insight into the complex biology of miRNA dependent processes.
\end{abstract}

Results: Here we analyze the contribution of microRNA-dependent transcriptional control in Pax8-expressing epithelial cells. Pax8 is a transcription factor that is crucial to the development of epithelial organs. The miRNA machinery was disrupted by crossing conditional DiGeorge syndrome critical region 8 (Dgcr8) fl/fl mice to Pax8Cre mice. The Dgcr8/ Drosha complex processes pri-miRNAs in the nucleus before they are exported as pre-miRNAs for further maturation by Dicer in the cytoplasm. Dgcr8 fl/fl; Pax8Cre+ knockout mice died prematurely, developed massive hypothyroidism and end stage renal disease due to a loss of miRNAs in Pax8 expressing tissue.

Conclusion: Pax8Cre-mediated conditional loss of DiGeorge syndrome critical region 8 (Dgcr8), an essential component of the nuclear machinery that is required for microRNA biogenesis, resulted in severe hypothyroidism, massively reduced body weight and ultimately led to renal failure and death of the animals. These data provide further insight into the importance of miRNAs in organ homeostasis using a Dicer independent model.

Keywords: Dgcr8, Cystic kidney disease, Kidney, MiRNA, Thyroid gland, CAKUT, Hydronephrosis, Pax8, Dicer

\section{Background}

The $\operatorname{Pax} 8$ gene encodes for a transcription factor that has been shown to play a pivotal role in the development of epithelial organ structures $[1,2]$. Expression of crerecombinase driven by the promoter of $\operatorname{Pax} 8$ has been shown in both kidney epithelia as well as the thyroid

\footnotetext{
*Correspondence: roman-ulrich.mueller@uk-koeln.de

${ }^{4}$ Systems Biology of Ageing Cologne, University of Cologne, Cologne, Germany

Full list of author information is available at the end of the article
}

gland. Pax8 itself is a transcription factor important in embryogenesis of the thyroid, Müllerian, and renal/upper urinary tracts. Consequently, a Pax 8 Cre mouse line is the perfect tool to address the importance of microRNAs (miRNAs) in the development and maintenance of two important epithelial organs-thyroid gland and kidneythrough targeted ablation of miRNA biogenesis.

Regulation of gene transcriptional networks by small non-coding RNAs (miRNAs) has been shown to play a crucial role in epithelial morphogenesis and the 
development and physiology of epithelial organs. Hence, it is not surprising that loss of microRNAs through conditional knockout of Dicer in the thyroid gland using the Pax 8 Cre mouse line resulted in the loss of thyroidal hormone production and death soon after weaning [3, 4]. However, these studies left some major questions unanswered. First, Dicer is not only involved in miRNA biogenesis, but also plays a role in other cellular processes such as genomic DNA fragmentation during apoptosis, the processing of endogenous siRNA and the detoxification of repeat elements [5-8]. Furthermore Dicer has recently been shown to bind to other RNA classes such as tRNAs, snoRNAs, mRNAs and promoter RNAs [9]. In this context Dicer binding does not necessarily involve cleavage but can also primarily impact on RNA-stability and recruitment of RNA molecules to p-granules. One way to confirm a phenotype observed in Dicer knockout animals to be truly dependent on miRNAs is confirmation of the phenotype knocking out a different component of the miRNA biogenesis machinery such as Dgcr8 $[10,11]$. Dgcr8 is the interaction partner of the RNAse 3 Drosha and as such essential to the maturation of most miRNAs. This has been done successfully before, e.g. in studies addressing the role of miRNAs in skin development or podocyte maintenance [12,13]. Dgcr8 itself has a number of functions independent from miRNA biogenesis as well $[14,15]$. However, an equal or at least highly similar phenotype between Dicer and Dgcr8 loss of function models would strongly reinforce the hypothesis of the phenotype to be miRNA-dependent since miRNA biogenesis is the only overlapping function between these two proteins known so far.

As to the kidney the specified anatomy of its epithelial composition adds another layer of complexity. In humans each kidney consists of about one million functional units-so called nephrons-that are made up by several specified segments each composed of a number of specialized epithelial cell types. Filtration of the blood occurs in the glomerulus thereby producing the primary urine. The urine is then processed in the tubulus system that can again be divided into several segments each fulfilling a different subset of functions regarding reabsorption and excretion of fluid and electrolytes. These differences in the properties of the tubulus cells along the nephron explain the distinct phenotypes that were described so far in regard to interference with miRNA processing by deleting Dicer conditionally in different segments of the tubulus system ranging from no obvious defects at all to rapid end-stage renal disease. For example, Dicer was deleted from the entire developing nephron lineage (Six2Cre), proximal tubulus (PEPCK-Cre), in the distal tubulus, collecting duct and developing ureteric bud (KspCre) or Wolffian duct, ureter, collecting duct and ureteric bud (HoxB7Cre) [16-21].

\section{Results}

To analyse the role of miRNAs in the development and maintenance of two epithelial organs - thyroid gland and kidney-and to understand to which extent the phenotype observed in Dicer-Pax8Cre knockout mice is indeed due to a lack of miRNAs we generated Dgcr8-Pax8Cre knockout mice (afterwards named Dgcr8 knockout).

D gcr $8 \mathrm{fl} / \mathrm{fl}$, Pax $8 \mathrm{Cre}+$ offspring was approximately born at the expected mendelian ratio (Additional file 1: Figure S1A). Nonetheless, starting already during the weaning period these mice were smaller and weighing showed a significantly decreased body weight in comparison to littermate controls (Fig. $1 \mathrm{a}, \mathrm{b}$ ). Analysis of free thyroxine (fT4) revealed massive hypothyroidism in Dgcr8 knockout mice since essentially almost no fT4 was detectable anymore (Fig. $1 \mathrm{c}$ ). The thyroid glands of $D$ gcr 8 knockout mice were considerably smaller than the controls. The follicular architecture was almost destroyed, few follicular structures were present with variable diameter and irregular outlines (Fig. $1 \mathrm{~d}$ ).

Molecular analysis of differentiation markers on knockout thyroid glands showed that the expression of the early differentiation markers Pax8, Nkx2.1 and Foxe1 is retained in the residual follicular cells, as well as the expression of their target Nis. Conversely, the expression of another late differentiation marker-Tg-resulted strongly reduced in the residual follicles. No alterations in calcitonin secreting cells were observed (Fig. 2). Thus, Dgcr8 knockout mice show functional defects partly similar to those from Dicer knockout mice [3, 4]. The main difference is that Nis is still expressed in Dgcr8 knockout mice, while being completely absent in Dicer knockout animals.

This severe hypothyroidism would generally be enough to explain the lethality of loss of microRNAs in the thyroid gland. However, as mentioned above previous studies showed that death in mice with a Pax8Cre mediated loss of Dicer in the thyroid gland could not be prevented by thyroxin substitution. This points to an additional mechanism leading to premature death in Pax8Cre mediated coditional loss of miRNAs [3].

Beside the thyroid gland it is known that $\operatorname{Pax} 8$ is also expressed in the kidney, especially in almost all segments of the kidney tubulus system [22].

Kidneys of the $\operatorname{Dgcr} 8$ knockout mice were smaller and weighed less than the control kidneys but there was no significant difference in the kidney/body weight ratio. Serum urea and serum creatinine levels were substantially increased in $\mathrm{Dgcr} 8-\mathrm{Pax} 8 \mathrm{Cre}$ knockout mice showing that these mice develop end-stage renal disease at the age of 4-6 weeks (Fig. 3 a, b). Quantitative PCR analysis confirmed the knockout of Dgcr8 in the kidney (Additional file 1: Figure S1B). 
a
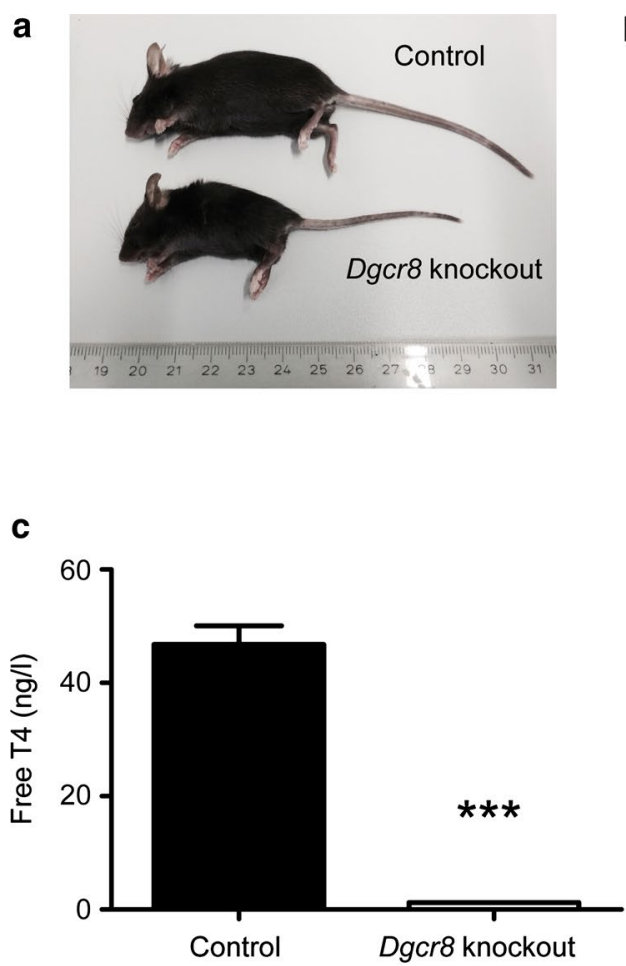

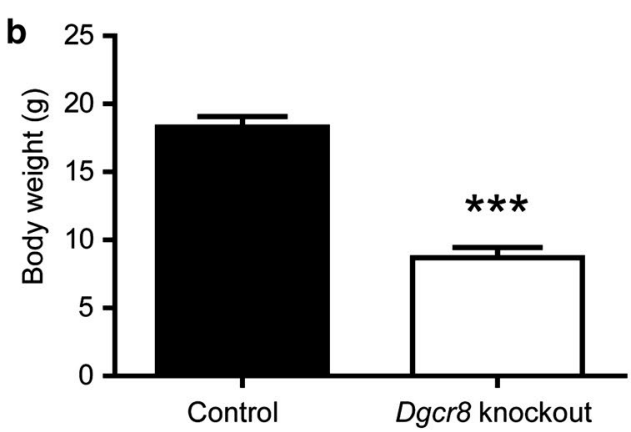

d

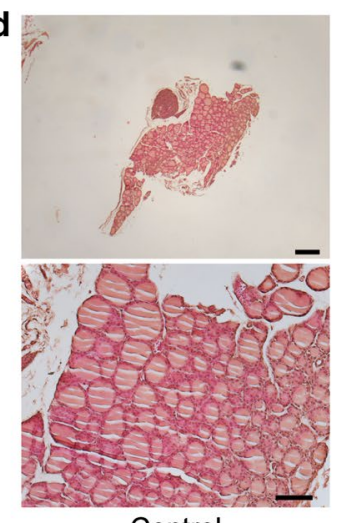

Control

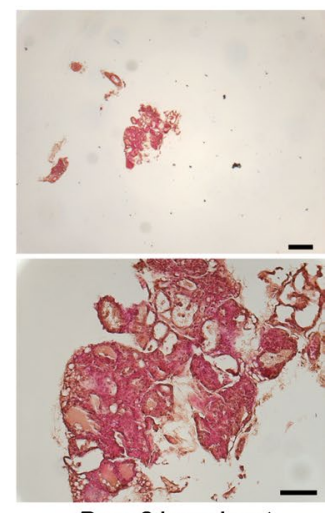

Dgcr8 knockout

Fig. 1 Dgcr8-Pax8Cre knockout animals develop severe hypothyroidism. a and $\mathbf{b}$ Dgcr8 knockout mice are smaller and have a reduced body weight (control $n=11$; knockout $n=9$; age 4-5 weeks; $p<0.0001$, error bars $=S E M$ ). $\mathbf{c}$ Dgcr8 knockout mice develop hypothyroidism as demonstrated by dramatically decreased fT4 levels in comparison to control littermates (control $n=5$; knockout $n=4$; age 4-8 weeks; $p<0.0001$, error bars = SEM). $\mathbf{d}$ H\&E staining of Dgcr 8 knockout thyroid glands reveals a severely disorganized follicular architecture (top panel $5 \times$ magnification, bar $100 \mu \mathrm{m}$; bottom panel 20x magnification, bar $50 \mu \mathrm{m}$ )

In line with this finding, histological analysis of the knockout kidneys revealed severe alterations in all parts of the tubulus system. We could detect hydronephrosis, proximal and distal tubular cysts, glomerular cysts and activated Bowman's epithelium. Also a strongly decreased parenchymal mass was detectable. (Fig. 4 a, b and Additional file 2: Figure S2).

Since $D g c r 8$ is one of the key components of efficent miRNA biogenesis we analysed the abundance of several miRNAs that have been reported to be enriched in kidney epithelial cells $[16,17]$. The expression of miR-192, miR-200b and miR-204 was significantly decreased in kidneys of $D g c r 8$ knockout mice (Fig. 5).

To further elucidate the consequences of loss of $D g c r 8$ to cellular biology in the kidney, we performed TUNEL assays. These stainings revealed a massive induction of apoptosis in all segments of the kidney (Fig. 6 a), suggesting that $D g c r 8$ dependent miRNAs are essential for the development and maintenance of a functional kidney tubulus system. Interestingly staining for the proliferation marker Ki-67 showed that beside apoptosis proliferation is also increased in the tubulus system, especially in the cortical region of $D g c r 8$ knockout mice pointing towards a general increase in cellular turnover (Fig. 6 b, c). Staining of thyroid gland tissue revealed that this increase of apoptosis (determined by TUNEL assays) on the one hand and proliferation (Ki-67 staining) on the other hand is not limited to the kidney but a general finding in the affected epithelial tissue of Dgcr8-Pax8Cre knockout mice (Additional file 3: Figure S3).

\section{Discussion}

We used the Pax8Cre-Allele to induce a conditional knockout of $D g c r 8$. The consequential loss of miRNAs in epithelial cells of the kidney and thyroid gland strongly perturbed the epithelial architecture of these organs leading to massive thyroid hypoplasia and dysplastic kidneys. On the functional side this phenotype is accompanied by not only severe hypothyroidism but also end-stage renal disease leading to premature death of the affected mice. Our mouse model highlights the importance of microRNAs to development and function of two relevant epithelial organs. Just like for most organs the general role of microRNAs in thyroid homeostasis and development 


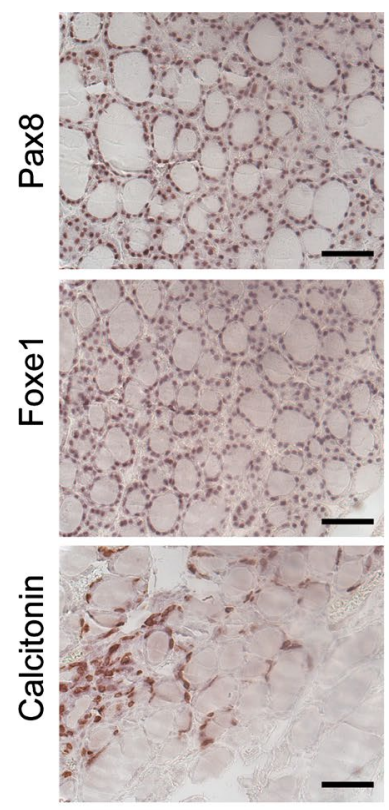

Control

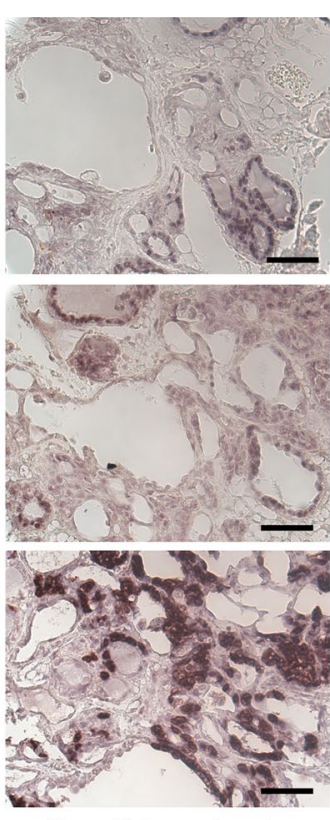

Dgcr8 knockout
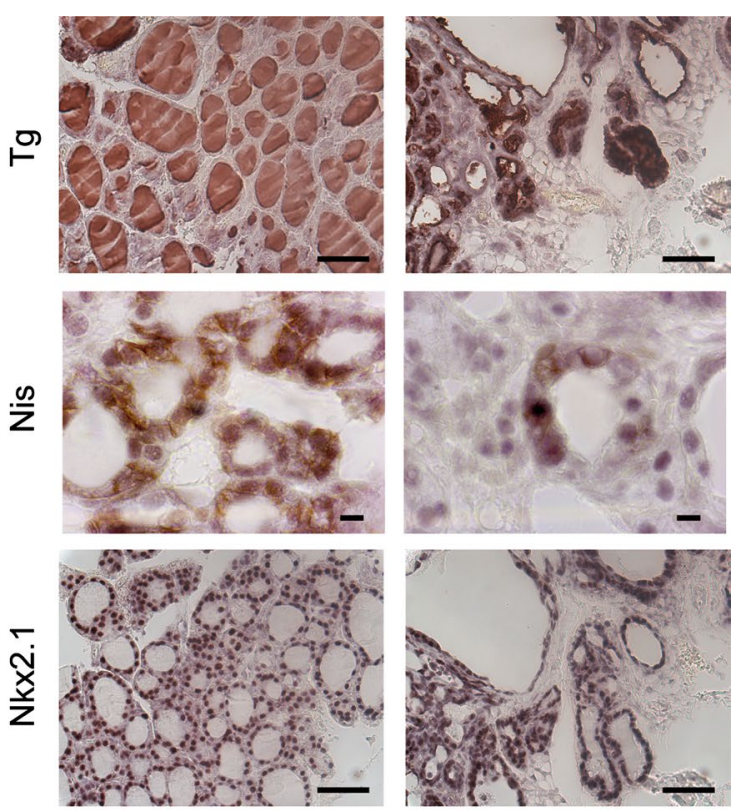

Control

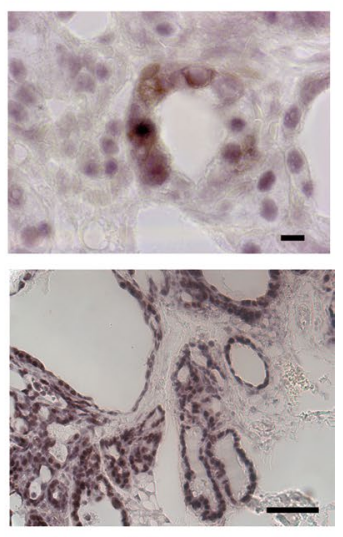

Dgcr8 knockout

Fig. 2 Analysis of differentiation markers in Dgcr8-Pax8Cre knockout thyroid glands. Immunohistochemistry of thyroid differentiation markers show a strong reduction of Tg expression. Pax8, Nkx2.1 and Foxe1 expression levels are unaffected in the residual follicular cells, as well as the expression of their target Nis. No alteration in calcitonin cells was observed. (40x magnification for Tg, Pax8, calcitonin, Nkx2.1 and Foxe1 bar 50 um; $100 \times$ magnification for Nis, bar $10 \mu \mathrm{m}$, age of the mice $4-8$ weeks)
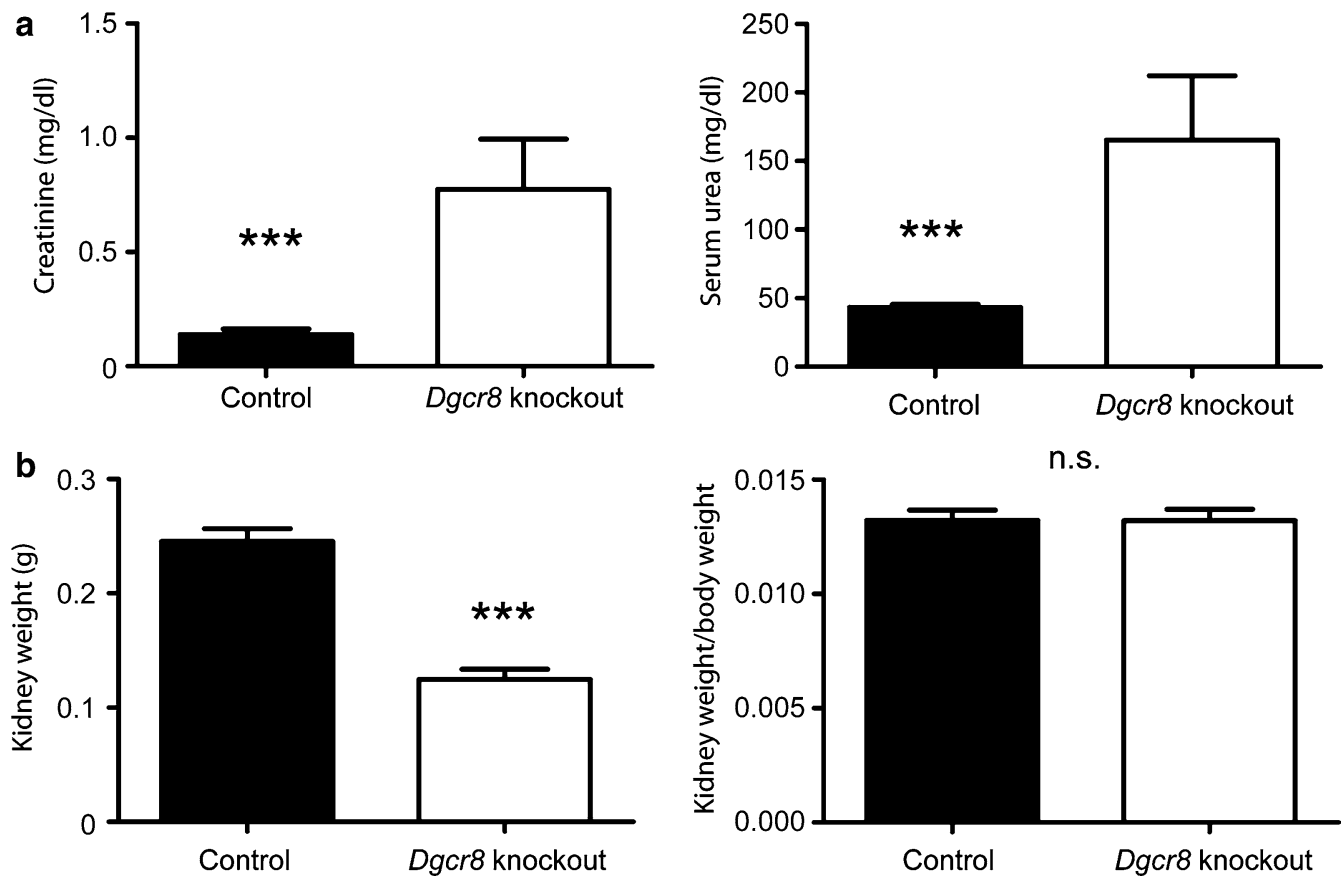

Fig. 3 Dgcr8-Pax8Cre knockout animals develop end stage renal disesase. a Dgcr8 knockout mice develop severe renal failure as shown by increased serum urea (knockout $n=4$, control $n=10 ; p<0$. 0005; error bars $=$ SEM) and serum creatinine (knockout $n=4$, control $n=10 ; p<0$. 0009; error bars $=$ SEM) levels. $\mathbf{b}$ The kidneys of Dgcr8-Pax8Cre knockout mice weigh less than control kidneys (knockout $n=10$, control $n=13$; $\mathrm{p}<0.0001$; error bars $=\mathrm{SEM}$ ) but there is no significant difference in the kidney/body weight ratio between these groups 
a

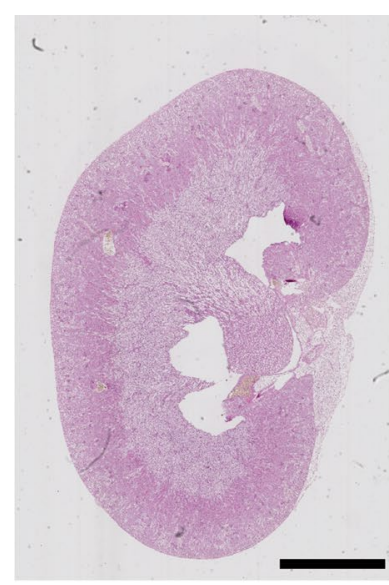

Control

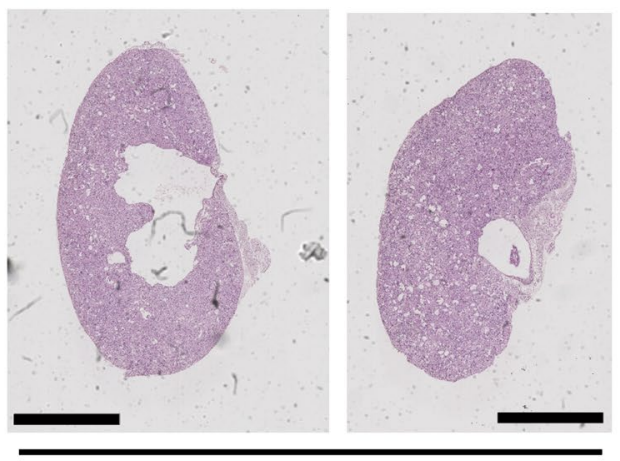

Dgcr8 knockout

b

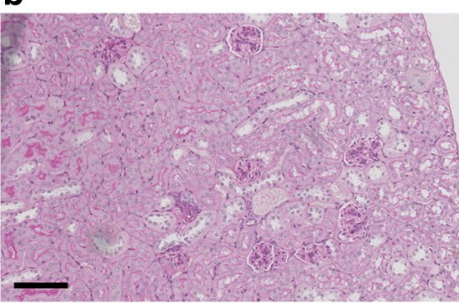

Control
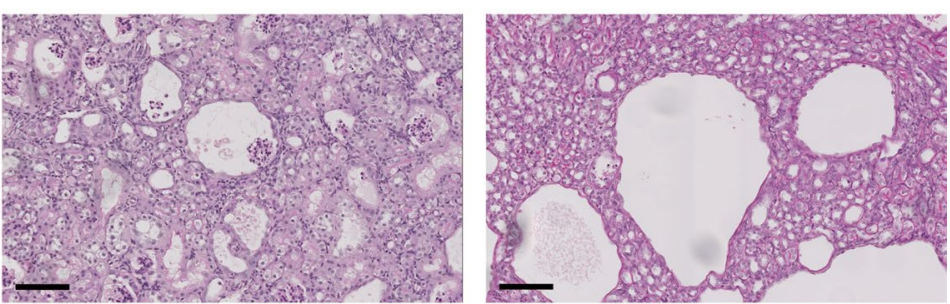

Dgcr8 knockout

Fig. 4 Dgcr8-Pax8Cre knockout mice have hydronephrotic and cystic kidneys. $\mathbf{a}$ and $\mathbf{b}$ PAS staining of the kidneys reveals alterations in all segments of the tubulus system. Beside a general reduction in kidney parenchyma (a), there are signs of hydronephrosis, cystic lesions in all parts of the nephron (b, glomerulocystic disease middle panel, tubular dilations/cysts right panel) and a marked activation of the Bowman epithelium (b, middle panel, see also Additional file 2: Figure S2) (a bar $2 \mathrm{~mm} ; \mathbf{b}$ bar $=100 \mu \mathrm{m}$, age of the mice 4-8 weeks)
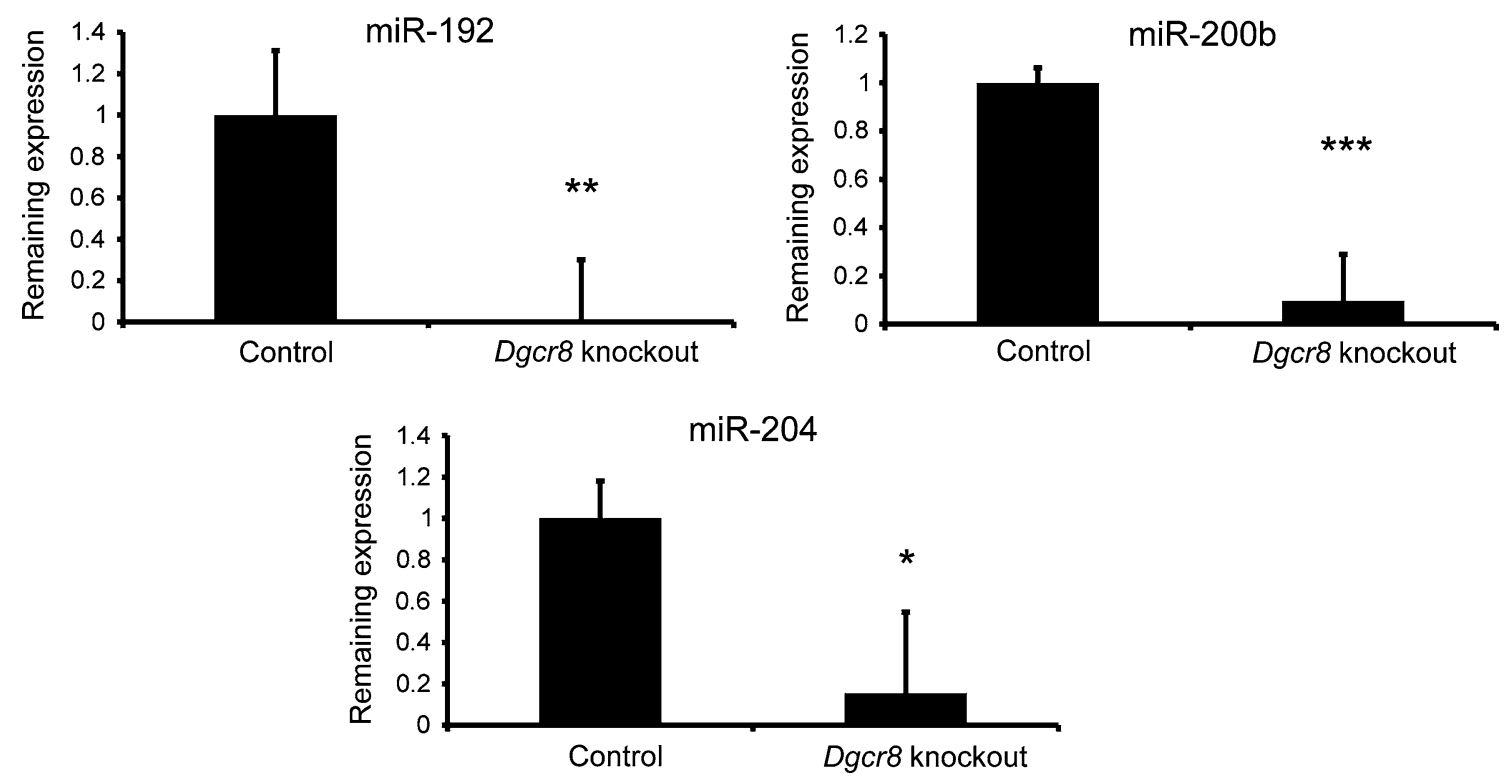

Fig. 5 Decreased expression of kidney specific miRNAs. Using qPCR the expression of several miRNAs was analysed. In line with a key role of Dgcr8 in miRNA processing, the kidney epithelial cell enriched miRNAs miR-192, miR-200b and miR-204 are strongly downregulated. SnoRNA-135 served as endogenous control $\left(n=4\right.$ per group, error bars $=S E M,{ }^{*} p<0.05,{ }^{* *} p<0.01,{ }^{* * *} p<0.001$, age of the mice $4-8$ weeks) 
a
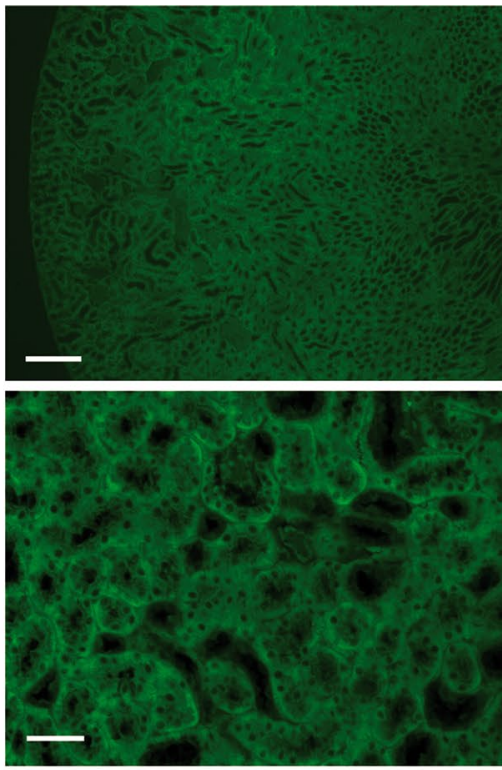

Control

b

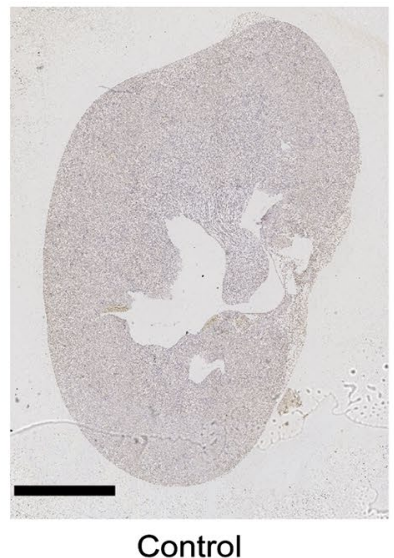

Control

C
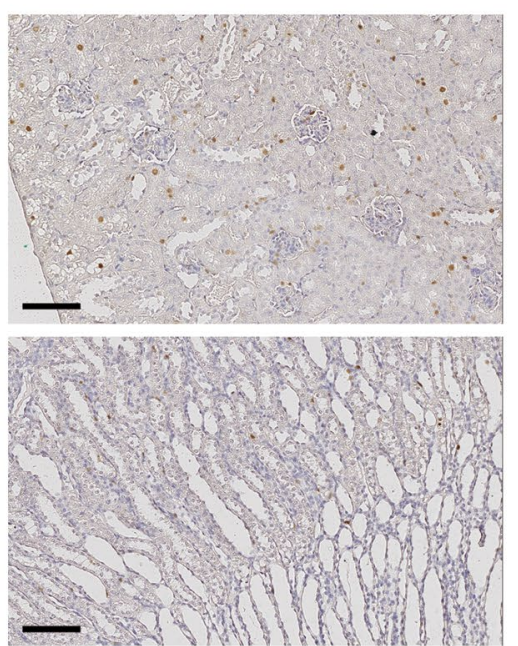

Control
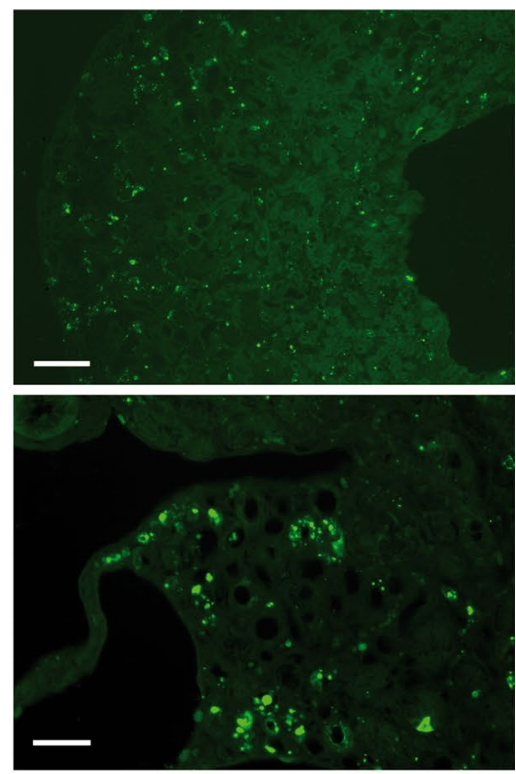

Dgcr8 knockout

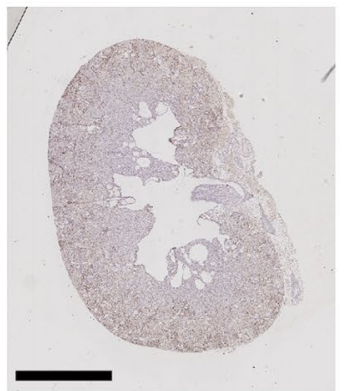

Dgcr8 knockout
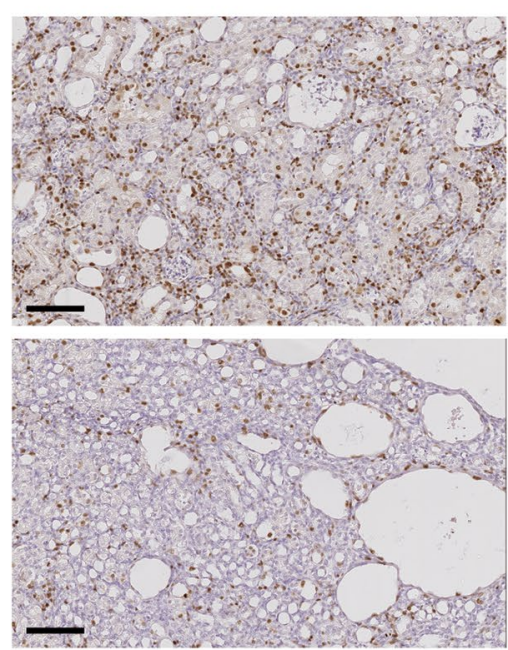

Dgcr8 knockout 
(See figure on previous page.)

Fig. 6 Dgcr8-Pax8Cre knockout kidneys show increased proliferation and apoptosis. a TUNEL staining reveals a massive upregulation of apoptosis in kidneys of Dgcr8 knockout mice (upper panel bar $200 \mu \mathrm{m}$; lower panel bar $50 \mu \mathrm{m}$, age of the mice 4-8 weeks). $\mathbf{b}$ and $\mathbf{c}$ An increase in proliferation is detectable especially in the cortex of Dgcr8 knockout kidneys (b bar 2 mm; c bar $100 \mu \mathrm{m}$ )

had been addressed using a conditional knockout of Dicer [3, 4]. Here we show that knockout of $D g c r 8$ leads to a similar phenotype of severe hypothyroidism due to thyroid hypoplasia confirming the importance of miRNAs for thyroid development and maintenance. Nonetheless we also observed some structural and molecular differences between Pax8Cre mediated loss of Dicer and $D g c r 8$. Although the general organization of the thyroid gland of $D g c r 8$ knockout mice is severely altered, some structures resembling functional follicles are still present, while Dicer knockout mice at the same age do not show any follicular structure. Residual follicles in $\mathrm{Dgcr} 8$ knockout mice show expression of Foxe1 and Pax8 transcription factors, however thyroglobulin is absent from lumina showing instead strong intracellular positivity in some cells. Moreover, $D g c r 8$ knockout mice still express the Nis iodide symporter, that resulted absent in Dicer knockout thyroids. We can thus speculate that, differently to what observed in Dicer knockout mice, the hypothyroidism observed in Dgcr8 knockout mice does not depend on a block of differentiation, as the differentiation markers $\mathrm{Tg}$ and Nis are expressed in the residual follicles. The inability of thyroid epithelial cells to form functional follicles could be instead due to defects in cell polarization, as suggested by the intracellular retention of Tg.

Whether the differences observed between these mouse models regarding the expression of thyroid differentiation markers are due to specific functions of the small number of microRNAs the processing of which depends only on either Dicer or Dgcr8 or is a consequence of entirely miRNA-independent functions of these proteins remains elusive as of now. Mechanistically seen this will be a highly interesting topic for further research.

The renal phenotype observed provides a good explanation why conditional Pax8Cre Dicer knockout mice could not be rescued by substitution of thyroxin, which would generally rescue the loss of thyroid function $[3,23$, 24]. Concomitant end-stage renal failure would inevitably lead to death in this setting. During preparation of this manuscript Iervolino et al. published the renal phenotype of Pax8Cre mediated knockout of Dicer, whichbeing largely in line with our findings-again strengthens our view that the phenotype observed is induced by the loss of microRNAs [20]. Renal hypoplasia and hydronephrosis in our mice is in line with the findings observed before regarding Dicer knockout using a number of different cre-alleles [16, 18]. Our findings in respect of cell death and proliferation allow for the hypothesis that this perturbed balance is the cell biological correlate of organ dysfunction upon global loss of microRNAs. This cell biological consequence of the loss of miRNAs is not only supported by other studies in different tissues [25-27]. We also found this phenotype in a KspCre mediated $D g c r 8$ knockout model [28]. As expected taking into account the complex epithelial organization of the kidney described above these mice show a phenotype distinct from the renal disease described here with much more pronounced hydronephrosis.

It will now be an exciting endeavor to clarify whether the dysregulation of cell proliferation and apoptosis is mediated by single miRNA sequences or are rather a consequence of the tremendous effects of a global loss of miRNA-mediated regulatory processes.

\section{Conclusion}

MiRNAs are important regulators of key processes on a cellular, tissue and organismal level. Here we show, that disruption of the miRNA processing machinery using a Dgcr8 dependent knockout in Pax8 expressing cells leads to a severe phenotpye in two epithelial organs that rely on the Pax8 transcription factor during its development. The knockout mice develop severe hypothyroidism due to thyroid gland hypoplasia and develop end stage renal disease with hydronephrosis, cystic kidney disease and hypoplastic kidneys. Consequently this study adds to the evidence of miRNAs being crucial to the normal function of Pax8-expressing epithelial cells. Furthermore the endstage renal disease observed provides an explanation why thyroid hormone substitution did not rescue mice with a Pax8Cre driven conditional knockout of Dicer in previous studies.

\section{Methods \\ Mice}

$D g c r 8 \mathrm{fl} / \mathrm{fl}$ mice were described previously [11] and provided by Elaine Fuchs (Rockefeller University, NY, USA). The Pax8Cre mouse line was a kind gift of Meinrad Busslinger (IMP, Vienna, Austria) [22]. Animals were housed in standardized specific pathogen-free conditions in the animal facility of the CMMC and CECAD (University of Cologne). Animals were fed ad libitum using standard mouse chow. Both female and male mice were used in the experiments. Genotyping was performed 
according to standard protocols as described previously (Dgcr8 fp: 5' - GACATCAATCTGAGTAGAGACAGG, Dgcr8 rp: 5' - CAGATGGTAACTAACCTGCCAACC, $D g c r 8$ wt allel $=244 \mathrm{bp}$, conditional $D g c r 8$ allel $=370 \mathrm{bp}$; Cre fp: 5' - GCA TAA CCA GTG AAA CAG CAT TGC TG, Cre rp: 5' - GGA CAT GTT CAG GGA TCG CCA GGC G, beta globin fp: 5' - TGC TCA CAC AGG ATA GAG AGG GCA GG, beta globin rp: 5'- GGC TGT CCA AGT GAT TCA GGC CAT CG, Cre allel $=269$ bp, beta globin (serves as internal control for the Cre genotyping) $=494 \mathrm{bp}$ ). All animal procedures were performed according to European (EU directive 86/609/EEC), national (TierSchG), and institutional guidelines. All procedures as well as the specific study presented here were approved by local governmental authorities (Landesamt für Natur, Umwelt und Verbraucherschutz NordrheinWestfalen, LANUV NRW). For the experiments, mice were anesthetized using isoflurane and afterwards killed using the cervical dislocation method.

\section{Histology}

Kidneys of the indicated animals were fixed in formalin, embedded in paraffin and stained with PAS according to standard protocols. To analyse the expression of Ki-67, slides of fixed and paraffin-embedded mouse kidneys were de-paraffinized using Xylol and descending concentrations of ethanol. Antigen retrieval was carried out by warming kidney slides in citrate buffer $(10 \mathrm{mM}, \mathrm{pH} 6)$ for 10 min using a microwave. After blocking with $3 \% \mathrm{H}_{2} \mathrm{O}_{2}$ and Avidin and Biotin (Vector Laboratories, Burlingame, CA, USA) for 15 min each, slides were sequentially incubated with the Ki-67 antibody (rabbit Ki-67 ab16667, abcam, 1:500 dilution, overnight at $4{ }^{\circ} \mathrm{C}$ ) and after washing with PBS with biotinylated anti-rabbit IgG (Jackson ImmunoResearch, West Grove, PA, USA; $1 \mathrm{~h}$ at room temperature). Kidney slides were labelled with $\mathrm{ABC}$ kit (Vector Laboratories, Burlingame, CA, USA), and development was carried out using diaminobenzidine solution (Sigma Aldrich, St. Louis, MO, USA). Slides were counterstained with hematoxylin (Sigma Aldrich, St. Louis, MO, USA), dehydrated and afterwards mounted with Histomount (National Diagnostics).

Stained slides were scanned using a Slidescanner (Leica, Wetzlar, Germany) and analysed using the ImageScope software (version 12.0.1.5030, Aperio).

Thyroids were fixed overnight at $4{ }^{\circ} \mathrm{C}$ in $4 \%$ paraformaldehyde in PBS, pH 7.2, dehydrated through ethanol series, cleared in xylene and embedded in paraffin. For histological analysis, $7 \mu \mathrm{m}$ sections were stained with hematoxylin and eosin (Sigma-Aldrich, St. Louis, MO, USA), according to the manufacturer's instructions. For immunohistochemical analysis, $7 \mu \mathrm{m}$ sections were dewaxed by standard techniques (Frezetti et al. 2011). The following primary antibodies were used at the indicated concentrations/dilutions: anti-Pax8 1:500 [29]; anti-Nkx2.1 1:3000 [30]; anti-Foxe1 1:500 [31]; anti-Nis 1:500 [32], anti-Calcitonin rabbit polyclonal antibody 1:2500 (Dako, Milan, Italy), anti-Thyroglobulin rabbit polyclonal antibody 1:500 (Dako), Biotinylated anti-rabbit IgG 1:200 was used for detection of primary antibodies (Vector Laboratories, Burlingame, CA, USA).

\section{TUNEL assay}

To evaluate apoptosis in the kidney we used the Promega DeadEnd Fluorometric Kit according to the manufacturers protocol (Promega, Madison, Wisconsin, USA). Pictures were taken with an inverted microscope (Axiovert200, equipped with an ApoTome system and an AxioCam MRm camera. Objectives used: Plan Apochromat $20 \times / 0.8$ NA and EC Plan Neofluar $5 \times /$ 0,16 NA. All from Carl Zeiss, Jena, Germany) using Axiovision 4.8 software for acquisition and subsequent image processing (Carl Zeiss, Jena, Germany).

\section{QPCR}

RNA was extracted from whole mouse kidneys using the phenol-chloroform extraction method. RT reactions were performed using the Taqman microRNA Reverse Transcription Kit (Life Technologies, Carlsbad, California, USA). Expression of miR-192 (assay ID 000491), miR-204 (assay ID 000508) and miR200b (assay ID 4426961) was analysed with Taqman assays (Life Technologies, Carlsbad, California, USA), snoRNA135 (assay ID 001230) served as endogenous control. To evaluate the expression level of Dgcr8 in the kidney of control and Dgcr8-Pax8Cre knockout mice, after RNA extraction from the whole kidney the RT reaction was performed using the high-capacity cDNA Kit (Life Technologies, Carlsbad, California, USA). The expression level of Dgcr8 (assay ID Mm.PT.58.33285106, Integrated DNA Technologies, Leuven, Belgium) was normalized against three endogenous controls (Gapdh, assay ID Mm 99999915_g1; Actb, assay ID Mm 02619580_g1; both from Life Technologies, Carlsbad, California, USA; and Hprt, assay ID Mm.PT.58.32092191, Integrated DNA Technologies, Leuven, Belgium). All qPCR experiments were performed on the ABI 7900HT System. Data analysis and statistics were performed using the delta-delta $\mathrm{Ct}$ method and a two tailed Student's t test.

\section{Laboratory medicine}

Blood was obtained by cardiac puncture. Plasma was prepared by centrifugation at $3000 \mathrm{rpm}$ for $10 \mathrm{~min}$. Urea, 
creatinine and fT4 was measured in the central laboratory medicine unit of the University Hospital of Cologne using the kinetic UV test (urea, Roche Diagnostics), creatinine plus test vers.2 (creatinine, Roche Diagnostics) and fT4 II test (fT4, Roche Diagnostics). Significance was calculated using a two-tailed Student's t test for all measurements.

\section{Additional files}

Additional file 1: Figure S1. Dgcr8-Pax8Cre mice are born at the Mendelian ratio and reduced expression level of Dgcr8 in Dgcr8-Pax8Cre knockout kidneys. A Dgcr8-Pax8Cre knockout mice are approximately born at the expected Mendelian ratio ( $n=50$ animals). $B$ The expression level of Dgcr8 was examined using qPCR ( $n=4$ animals per group, error bars $=\mathrm{SEM}^{* * *} \mathrm{p}<0.001$ ). Since whole kidneys were subjected to the analysis, but conditional knockout is limited to Pax8 expressing cells the remaining expression of Dgcr8 is about $50 \%$ in comparison to the control group.

Additional file 2: Figure S2. Additional histological images of Dgcr8 knockout kidneys. PAS staining of the kidneys of Dgcr8 knockout mice shows cysts in the collecting duct, distal and proximal tubulus system, glomerular cysts with activated Bowman epithelium and a reduction in kidney parenchym (bar $=100 \mu \mathrm{m})$.

Additional file 3: Figure S3. Increased levels of apoptosis and proliferation in thyroid glands of Dgcr8-Pax8Cre knockout mice. A An increase in apoptotic cells in the thyroid gland of Dgcr8 knockout animals were detected by TUNEL assays (bar $=100 \mu \mathrm{m}$ ). B Similar to the kidney, there is a marked increase in proliferation as detected by Ki-67 stainings in the thyroid gland of Dgcr8 knockout mice (bar $=100 \mu \mathrm{m})$

\section{Abbreviations}

CAKUT: congenital anomalies of the kidney and urinary tract; Dgcr8: DiGeorge syndrome critical region 8; miRNA: microRNA; Pax8: paired box 8; Nis: sodium iodide symporter; Tg: thyroglobulin; $\mathrm{fT} 4$ : free thyroxin.

\section{Authors' contributions}

Conceived and designed experiments: MPB, EA, TB, BS, GV and RUM. Performed experiments: MPB, EA, RUM. Analysed data: MPB, EA, GV, RUM. Wrote the paper: MPB, EA, TB, BS, GV, RUM. All authors read and approved the final manuscript.

\section{Author details}

${ }^{1}$ Department II of Internal Medicine and Center for Molecular Medicine, University of Cologne, Kerpener Str. 62, 50937 Cologne, Germany. ${ }^{2}$ Dipartimento di Biologia e Patologia Cellulare e Molecolare, Università degli Studi di Napoli 'Federico II', Naples, Italy. ${ }^{3}$ Cologne Excellence Cluster on Cellular Stress Responses in Aging-Associated Diseases, University of Cologne, Cologne, Germany. ${ }^{4}$ Systems Biology of Ageing Cologne, University of Cologne, Cologne, Germany.

\section{Acknowledgements}

We thank Elaine Fuchs and Meinrad Busslinger for providing the Dgcr8 fl/ $f l$ and Pax 8 Cre mouse lines, respectively and members of the laboratory for helpful discussion. We would like to thank Martyna Brütting and Kathrin Riehl for excellent technical assistance. We thank the CECAD Imaging Facility for technical support with the Slidescanner.

\section{Availability of data and materials}

All the data supporting our findings is contained within the manuscript or in the supplementary material. Raw data images from the microscopy work as well as regarding the $\mathrm{qPCR}$ experiment are available upon request.

\section{Competing interests}

The authors declare that they have no competing interests.

\section{Ethics}

All animal procedures were performed according to European (EU directive 86/609/EEC), national (TierSchG), and institutional guidelines. All procedures as well as the specific study presented here were approved by local governmental authorities (Landesamt für Natur, Umwelt und Verbraucherschutz Nordrhein-Westfalen, LANUV NRW, Germany). Approval by the LANUV includes the review and approval by an ethics committee.

\section{Funding information}

This work was funded by the "Deutsche Nierenstiftung" to RUM, by the "Deutsche Forschungsgemeinschaft" SFB829 to TB and SCHE1562-2 to BS. GDV and EA are supported by a grant from "Associazione Italiana per la Ricerca sul Cancro".

Received: 30 October 2015 Accepted: 7 April 2016

Published online: 18 April 2016

\section{References}

1. Lang D, Powell SK, Plummer RS, Young KP, Ruggeri BA. PAX genes: roles in development, pathophysiology, and cancer. Biochem Pharmacol. 2007;73:1-14.

2. Mansouri A, Hallonet M, Gruss P. Pax genes and their roles in cell differentiation and development. Curr Opin Cell Biol. 1996;8:851-7.

3. Rodriguez W, Jin L, Janssens V, Pierreux C, Hick A-C, Urizar E, et al. Deletion of the RNaselll enzyme dicer in thyroid follicular cells causes hypothyroidism with signs of neoplastic alterations. PLoS ONE. 2012;7:e29929.

4. Frezzetti D, Reale C, Calì G, Nitsch L, Fagman H, Nilsson O, et al. The microRNA-processing enzyme dicer is essential for thyroid function. PLoS ONE. 2011;6:e27648.

5. Johanson TM, Lew AM, Chong MMW. MicroRNA-independent roles of the RNase III enzymes Drosha and Dicer. Open Biol. 2013;3:130144.

6. Calabrese JM, Seila AC, Yeo GW, Sharp PA. RNA sequence analysis defines Dicer's role in mouse embryonic stem cells. Proc. Natl. Acad. Sci. USA. 2007; 104:18097-102.

7. Tam OH, Aravin AA, Stein P, Girard A, Murchison EP, Cheloufi S, et al. Pseudogene-derived small interfering RNAs regulate gene expression in mouse oocytes. Nature. 2008;453:534-8.

8. Babiarz JE, Ruby JG, Wang Y, Bartel DP, Blelloch R. Mouse ES cells express endogenous shRNAs, siRNAs, and other Microprocessor-independent dicer-dependent small RNAs. Genes Dev. 2008;22:2773-85.

9. Rybak-Wolf A, Jens M, Murakawa Y, Herzog M, Landthaler M, Rajewsky N. A variety of dicer substrates in human and C. elegans. Cell. 2014;159:1153-67.

10. Landthaler M, Yalcin A, TuschI T. The human DiGeorge syndrome critical region gene 8 and Its D. melanogaster homolog are required for miRNA biogenesis. Curr. Biol. 2004;14:2162-7.

11. Wang Y, Medvid R, Melton C, Jaenisch R, Blelloch R. DGCR8 is essential for microRNA biogenesis and silencing of embryonic stem cell self-renewal. Nat Genet. 2007;39:380-5.

12. Yi R, Pasolli HA, Landthaler M, Hafner M, Ojo T, Sheridan R, et al. DGCR8dependent microRNA biogenesis is essential for skin development. Proc Natl Acad Sci. 2009;106:498-502.

13. Zhdanova O, Srivastava S, Di L, Li Z, Tchelebi L, Dworkin S, et al. The inducible deletion of Drosha and microRNAs in mature podocytes results in a collapsing glomerulopathy. Kidney Int. 2011;80:719-30.

14. Luhur A, Chawla G, Wu Y-C, Li J, Sokol NS. Drosha-independent DGCR8/ Pasha pathway regulates neuronal morphogenesis. Proc Natl. Acad Sci USA. 2014;111:1421-6.

15. Macias S, Plass M, Stajuda A, Michlewski G, Eyras E, Cáceres JF. DGCR8 HITS-CLIP reveals novel functions for the microprocessor. Nat Struct Mol Biol. 2012;19:760-6.

16. Bartram MP, Höhne M, Dafinger C, Völker LA, Albersmeyer M, Heiss J, et al. Conditional loss of kidney microRNAs results in congenital anomalies of the kidney and urinary tract (CAKUT). J Mol Med Berl Ger. 2013;91:739-48.

17. Patel V, Hajarnis S, Williams D, Hunter R, Huynh D, Igarashi P. MicroRNAs Regulate Renal Tubule Maturation through Modulation of Pkd1. J Am Soc Nephrol JASN. 2012;23:1941-8. 
18. Nagalakshmi VK, Ren Q, Pugh MM, Valerius MT, McMahon AP, Yu J. Dicer regulates the development of nephrogenic and ureteric compartments in the mammalian kidney. Kidney Int. 2011;79:317-30.

19. Pastorelli L, Wells S, Fray M, Smith A, Hough T, Harfe B, et al. Genetic analyses reveal a requirement for Dicer1 in the mouse urogenital tract. Mamm Genome. 2009;20:140-51.

20. lervolino A, Trepiccione F, Petrillo F, Spagnuolo M, Scarfò M, Frezzetti $D$, et al. Selective Dicer suppression in the kidney alters GSK3 $\beta / \beta$ catenin pathways promoting a glomerulocystic disease. PLoS ONE. 2015;10:e0119142.

21. Wei Q, Bhatt K, He H-Z, Mi Q-S, Haase VH, Dong Z. Targeted deletion of dicer from proximal tubules protects against renal ischemia-reperfusion injury. J Am Soc Nephrol. 2010;21:756-61.

22. Bouchard M, Souabni A, Busslinger M. Tissue-specific expression of cre recombinase from the Pax8 locus. Genes NYN. 2000;2004(38):105-9.

23. De Felice M, Di Lauro R. Thyroid development and its disorders: genetics and molecular mechanisms. Endocr Rev. 2004;25:722-46.

24. De Felice $M$, Di Lauro R. Murine models for the study of thyroid gland development. Endocr Dev. 2007;10:1-14.

25. Harfe BD, McManus MT, Mansfield JH, Hornstein E, Tabin CJ. The RNasell enzyme Dicer is required for morphogenesis but not patterning of the vertebrate limb. Proc Natl Acad Sci USA. 2005;102:10898-903.

26. Shi S, Yu L, Chiu C, Sun Y, Chen J, Khitrov G, et al. Podocyte-selective deletion of dicer induces proteinuria and glomerulosclerosis. J Am Soc Nephrol. 2008;19:2159-69.
27. Pernaute B, Spruce T, Smith KM, Sánchez-Nieto JM, Manzanares M, Cobb $B$, et al. MicroRNAs control the apoptotic threshold in primed pluripotent stem cells through regulation of BIM. Genes Dev. 2014;28:1873-8.

28. Bartram MP, Dafinger C, Habbig S, Benzing T, Schermer B, Müller R-U. Loss of Dgcr8-mediated microRNA expression in the kidney results in hydronephrosis and renal malformation. BMC Nephrol. 2015;16:55.

29. Amendola E, De Luca P, Macchia PE, Terracciano D, Rosica A, Chiappetta $\mathrm{G}$, et al. A mouse model demonstrates a multigenic origin of congenital hypothyroidism. Endocrinology. 2005;146:5038-47.

30. Lazzaro D, Price M, de Felice M, Di Lauro R. The transcription factor TTF-1 is expressed at the onset of thyroid and lung morphogenesis and in restricted regions of the foetal brain. Dev Camb Engl. 1991;113:1093-104.

31. Dathan N, Parlato R, Rosica A, De Felice M, Di Lauro R. Distribution of the titf2/foxe1 gene product is consistent with an important role in the development of foregut endoderm, palate, and hair. Dev Dyn Off Publ Am Assoc Anat. 2002;224:450-6.

32. Postiglione MP, Parlato R, Rodriguez-Mallon A, Rosica A, Mithbaokar P, Maresca M, et al. Role of the thyroid-stimulating hormone receptor signaling in development and differentiation of the thyroid gland. Proc Natl Acad Sci USA. 2002:99:15462-7.

\section{Submit your next manuscript to BioMed Central and we will help you at every step:}

- We accept pre-submission inquiries

- Our selector tool helps you to find the most relevant journal

- We provide round the clock customer support

- Convenient online submission

- Thorough peer review

- Inclusion in PubMed and all major indexing services

- Maximum visibility for your research

Submit your manuscript at www.biomedcentral com/submit 